\title{
VARIAZIONE DI RESISTENZA DEL MERCURIO E DELLE AMALGAME DI BISMUTO NEL CAMPO MAGNETICO.
}

Nota del Dott. GaETano ROSSI $\left.{ }^{1}\right)$.

Usando correnti continue per la misura delle variazioni di resistenza: Neesen ${ }^{2}$ ) (1884) trovò che l'influenza di campi relativamente deboli causa diminuzione di resistenza nelle dissoluzioni di cloruro ferrico, solamente se disposte parallelamente alle linee di forza. Mentre Bagard ${ }^{3}$ ) (1889) osservò aumento dell' $1 \%$ per dissoluzioni di $\mathrm{CuSO}_{4}$ in campi di 5000 Gauss, la posizione essendo la equatoriale. Drude e Nernst ${ }^{4}$ ) (1891) trovarono aumento di $0,20 \%$ in campi di 8000 Gauss per l' $\mathrm{Hg}$ e di $0,4 \%$ o pel $\mathrm{Bi}$ fuso.

Usando corrente alternata, Berndt ${ }^{5}$ ) (1908) trovò invece per l' $\mathrm{Hg}$ e pel Bi fuso variazioni rispettivamente non superiori al $0,00005 \%$ ed al $0,004 \%$, variazioni che attribui ad azioni elettrodinamiche, mentre in campi da 1000 a 3000 Gauss trovò che l' influenza era nulla per soluzioni saline di $\mathrm{Fe}, \mathrm{Ni}, \mathrm{Co}, \mathrm{Bi}$.

Questi risultati che lasciano ancora insoluto il problema della variazione delle resistenze liquide nel campo magnetico indussero il Prof. Cantone a consigliarmi la ripresa in esame del fenomeno.

1) Lavoro eseguito nel Laboratorio di Fisica della R. Università di Napoli.

2) Wied Ann. 23, 482, 1884.

$\left.{ }^{3}\right)$ Comptes Rendus. T. $28^{\mathrm{me}}$. Juin 1899.

4) Wied. Ann. 42, 573, 1891.

s) Journal de Physic. Mars. 1908. 
Per la determinazione del campo magnetico utilizzai una elettrocalamita del Faraday; le facce polari misuravano cm. 8,46 di diametro e, rese perfettamente parallele, furono, durante le mie ricerche, serbate costantemente distanti fra loro $\mathrm{m} / \mathrm{m} .7,7 \overline{5}$ : il minimo possibile.

Per la misura dell' intensità del campo ricorsi al metodo dell' induttore terrestre, applicando la formola

$$
\mathrm{H}=h \frac{n^{\prime} \mathrm{S}^{\prime}}{n \mathrm{~S}} \cdot \frac{d}{d^{\prime}}
$$

dove $h$ è la componente orizzontale del magnetismo terrestre, determinata in questo laboratorio nell' anno 1907, e trovata uguale a 0,228 Gauss; $n^{\prime} \mathrm{S}^{\prime}$ ed $n \mathrm{~S}$ rappresentano rispettivamente le aree totali dell' induttore terrestre e della bobina di riferimento racchiusa nella elettrocalamita; $d^{\prime}$ è la deviazione, osservata sulla scala del galvanometro balistico, quando si fa rotare di $180^{\circ}$ l' induttore terrestre attorno ad un asse verticale, corretta per l' effetto dello smorzamento; e $d$ la deviazione, ugualmente corrretta, dovuta all' inversione rapida del campo,

L' intensità di detto campo si fece variare da 3350 a 4450 Gauss, corrispondentemente ad una corrente magnetizzante da 3 a 5 Ampères, fornita da 10 accumulatori.

Per la misura delle resistenze liquide utilizzai un ponte a filo del Kohlrausch e come strumento di zero un ottimo telefono Adler; però per la misura delle variazioni di resistenza, dovute al campo, non mi avvalsi dello stesso ponte, perchè, essendomi assicurato che anche colle correnti continue colle opportune cautele si possono avere risultati vantaggiosi, non volli rinunziare alle osservazioni galvanometriche, le quali, a mio giudizio, sono più attendibili e conferiscono alla disposizione una sensibilità maggiore che non le telefoniche.

Dirò intanto circa la costruzione delle resistenze liquide: usai tubi capillari di vetro di $\mathrm{m} / \mathrm{m} .0,7$ e m/m. 0,5 di diametro, piegati alla lampada come è indicato dalla fig. 1. Gli estremi $e$ di tali tubi, piegati a doppia squadra finivano col pescare ciascuno in un pozzetto $p$ contenente lo stesso liquido 
(mercurio o amalgama) che riempiva il tubo, e di diametro abbastanza grande perchè la resistenza ne fosse trascurabile. Nei pozzetti mettevano capo i fili $f$ di rame adduttori della corrente.

$$
\text { Fig.t }
$$

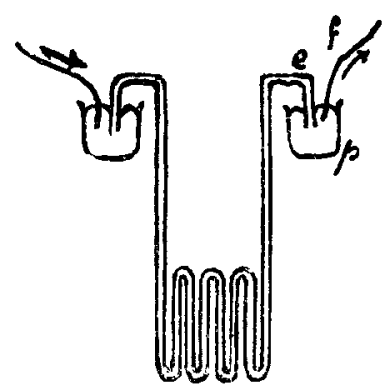

I tubi venivano per la maggior lunghezza incassati fra lastre di paraffina che, oltre a dar loro maggiore consistenza, solidita e perfetto isolamento, li proteggevano da repentine eventuali variazioni di temperatura producentisi nell'ambiente, o ritardavano la comunicazione del calore prodotto dall' effetto Joule dovuto alla corrente magnetizzante.

\section{Fig. 2}

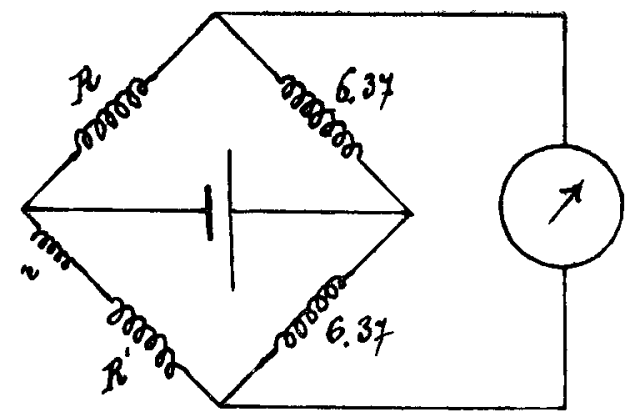

Per la misura delle variazioni di resistenza mi servii di una cassetta contenente 2 rocchetti di filo di manganina e- 
guali ciascuno in resistenza a $6,37 \mathrm{Ohm}$ e costituenti due lati contigui del ponte (fig. 2), mentre da rimanenti lati funzionavano due resistenze liquide $R$ ed $R$ ', eguali e di identica natura, costruite come ho detto.

Delle due resistenze liquide, quella in esame $R^{\prime}$ (protetta come dissi) veniva stretta tra le masse polari della elettrocalamita (fig. 3).

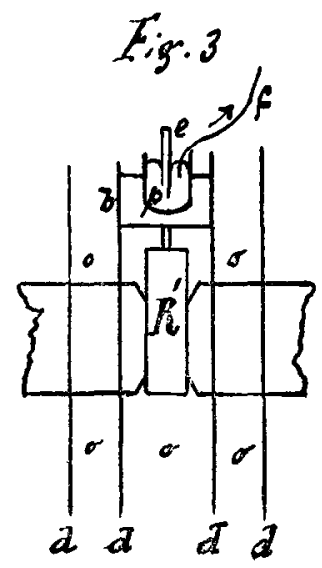

Quattro dischi di cartone $d$, ricoperti di stagnola, erano disposti come indica la figura, e, di questi i due più interni servivano di sostegno a due blocchetti $h$ di paraffina dove erano fissati i pozzetti ai quali terminavano gli estremi $e$ del tubo capillare.

I fili di rame $f$, adduttori della corrente, erano fissati invariabilmente ai pozzetti, $\dot{\theta} l^{\prime}$ intero sistema ora descritto era abbastanza rigido per garentirne la inamovibilità delle parti.

Dell' ovatta o riempiva i vani fra $i$ dischi, ed un coperchio di cartoncino paraffinato ricoperto prima di ovatta ed infine esternamente di stagnola proteggeva le parti scoperte $e$ del tubo.

Analoghe cautele furono adottate per la resistenza gemella $R$, che, lontana dalla elettrocalamita, sostenuta in un vaso di vetro ripieno di ovatta e bene isolato, riposava in 
una cassetta anch' essa ripiena di ovatta e dotata di coperchio di carta paraffinata.

Questa disposizione cosi accurata e simmetrica, in un ambiente, per quanto possibile a temperatura uniforme, garentiva la costanza della temperatura nelle due resistenze liquide, giacchè il calore che si svolgeva al passaggio della corrente nel ponte, benchè minimo, veniva ad accumularsi in egual misura nelle due resistenze egualmente protette.

Riuscii ad evitare in parte l' effetto Joule dovuto alla corrente magnetizzante usando l'avvertenza di non lavorare a lungo e ricorrendo a correnti non superiori a 5 Ampères, perchè dopo qualche tempo il calore, dalla elettrocalamita, superando le protezioni, passava alla resistenza $R$, e vi permaneva a lungo, come è facile capire, a causa delle stesse protezioni.

Curai inoltre un isolamento perfetto in tutte le parti della mia disposizione, usando paraffina in larga misura, e peranco costruendomi un tasto, in paraffina rame e mercurio, per la chiusura dei circuiti.

Nel lato in esame $R^{\prime}$ trovavasi di più intercalata una debolissima resistenza variabile $r$ che funzionava da regolatore, costituita nella parte sua essenziale da un grosso filo di rame immerso in mercurio, e nel quale poteva venire affondato o sollevato, subordinatamente al comando di una vite di $\mathrm{m} / \mathrm{m} .11 / 2$ di passo.

Il regolatore fu costruito con metalli non magnetici e fissato invariabilmente ad un tavolo di sostegno. Esso aveva un doppio ufficio: anzitutto permetteva di raggiungere la perfetta uguaglianza fra i lati $R, R^{\prime}$ del ponte, o di potervela ricondurre allorquando, pel debole calore, passato dalla elettrocalamita alla resistenza $R$, cessava; ed in secondo luogo dava modo di determinarmi la sensibilità del sistema.

Il metodo seguito nelle ricerche fu il seguente: realizzato il ponte come è indicato nella fig. 2, dove la corrente era fornita da una Daniell ed il galvanometro era un sensibilissimo Hartmanu \& Braun, procedevo così: servendomi del regolatore uguagliavo $i$ lati $R, R$ in modo da ottenere, chiudendo il circuito, una deviazione galvanometrica $a$ di poco 
inferiore al 500 (posizione di zero del galvanometro), indi aprivo detto circuito, eccitavo il campo, ed, a campo eccitato, richiudevo il circuito facendo una seconda lettura $b$; infine riaprivo il circuito, escludevo il campo, e tornava a chiuderlo, facendo una terza lettura $c$. Di $a$ e $c$, posizioni iniziale e finale del galvanometro senza del campo, pigliavo la media aritmetica $m$, e la differenza $\Delta=b-m$ dava la deviazione dovuta al campo.

Non toccando più il regolatore, per ciascun campo ripetevo 5 volte l'operazione suindicata: per effetto termico, lo 5 deviazioni successive iniziali $a$ procedendo progressivamente crescenti, io stabiliva la prima in modo che tra esse cadesse pressochè intermedia la posizione zero (500) del galvanometro (come può rilevarsi dalle tabelle I e II). È vero che col regolatore avrei potuto riportarmi sempre a zero, volta per volta, ma ciò importava, oltre alla perdita di tempo con relativa propagazione di calore alla resistenza nell' intraferro, un continuo disturbo del rassettamento del sistema.

Delle cinque $\Delta$ pigliavo poi la media.

Dirò ora circa il secondo ufficio del regolare: Avvalendomi di un ponte a cassetta Hartmann \& Braun, osservata la deviazione dovuta ad $\frac{1}{10000}$ di Ohm trovai il numero di giri, ai quali era obbligata la testa della vite del regolatore, perchè un certo tratto del filo di rame emergendo o immergen dosi nel mercurio producesse la stessa deviazione.

Potei così calcolare la resistenza, trovata eguale a $\frac{2}{100000}$ di Ohm, del tratto di filo di rame corrispondente ad un giro.

Ritornando alla disposizione del ponte (fig. 2): conosciuta la deviazione media $\Delta$ (di cui sopra) dovuta al campo e quella media $d$ dovuta ad un giro del regolatore, ossia a 2 $\frac{2}{100000}$ di Ohm, nel sistema in esame, era facile calcolare la variazione $v$ di resistenza che per effetto del campo subiva la frazione $\rho$ di resistenza liquida direttamente sottoposta al- 
l'azione del campo, e quindi determinare il rapporto $\delta$ tra la variazione $v$ e detta frazione di resistenza.

Ad evitare complicazioni di cifre decimali esprimo le $\delta$ in decimillionesimi.

Le mie ricerche si riferiscono all'Hg puro ed alle amalgame di $\mathrm{Bi}$, nel campo trasverso. Queste ultime, studiate ora, pare, per la prima volta, e da me prescelte a causa del notevolissimo aumento di resistenza che il Bi presenta nel campo magnetico, furono di quattro concentrazioni differenti: le prime due contenevano con 100 parti in peso di $\mathrm{Hg}$, rispettivamente parti 0,5 ed $1,0 \mathrm{di} \mathrm{Bi}$, le altre furono soluzioni sature di $\mathrm{Bi}$ in $\mathrm{Hg}$. a $12^{\prime}$ e $190^{\prime \prime}$ rispettivamente.

Tutte furono preparate nel vuoto, allo scopo d'impedire la formazione di ossido di $\mathrm{Bi}$, e poi obbligate a passare più volte per tubi affilatissimi a fine di privarle di particelle solide.

Le mie osservazioni furono eseguite con sole due coppie di tubi una da $\mathrm{m} / \mathrm{m} .0,7 \mathrm{l}$ 'altra da $\mathrm{m} / \mathrm{m}$. 0, reputo utile aggiungere che $\mathrm{i}$ detti tubi tutte le volte che vennero vuotati, per essere riempiti di amalgame più concentrate, presentarono sempre le pareti interne perfettamente terse.

Riporto le tavole relative al mercurio distillato ed alla amalgama di $\mathrm{Bi}$ satura a $190^{\circ}$, in tubo da m/m. 0, 


\section{Mercurio Distillato.}

Diametro del tubo $\mathrm{m} / \mathrm{m}$. 0,5 .

Resistenza totale $\mathrm{R}^{\prime}=\mathrm{R}=\frac{777}{223} \mathrm{\Omega}=3,48 \Omega$.

Parte sottoposta al campo $\rho=\frac{1}{2} \mathrm{R}^{\prime}=1,74 \Omega$

Deviazione per $\frac{2}{100000} \Omega, d=5,2$.

\begin{tabular}{|c|c|c|c|c|c|c|c|c|c|}
\hline \multicolumn{5}{|c|}{$H=3350$} & \multicolumn{5}{|c|}{$H=3640$} \\
\hline$a$ & $b$ & $c$ & $m$ & $\Delta$ & $a$ & $b$ & $c$ & $m$ & $\Delta$ \\
\hline 97,4 & 500,0 & 497,6 & 497,5 & $2, \check{5}$ & 488,6 & 492,9 & 491,0 & 489,8 & 3,1 \\
\hline 499,0 & 502,5 & 500,1 & 499,5 & 3,0 & 493,1 & 496,9 & 495,0 & 494,0 & 2,9 \\
\hline 501,0 & $\overline{\mathrm{o}} 0 \overline{\mathrm{o}, 0}$ & 504,0 & $502, \overline{0}$ & 2,5 & 496,1 & 500,4 & 497,9 & 497,0 & 3,4 \\
\hline $04, \tilde{\mathbf{z}}$ & $\tilde{0} 07,8$ & 505,8 & 505,1 & 2,7 & 503,0 & 506,5 & 503,9 & $\bar{\rho} 03, \overline{0}$ & 3,0 \\
\hline 506,0 & 509,0 & 507,2 & 506,6 & 2,4 & $50 \tilde{5}, 2$ & 509,0 & 507,0 & $\check{0} 06,1$ & 2,9 \\
\hline
\end{tabular}

Media $\Delta=2,6$

Media $\Delta=3,0$

$$
\mathrm{H}=3930
$$

$$
\mathrm{H}=4190
$$

\begin{tabular}{c|c|c||c|c}
$a$ & $b$ & $c$ & $m$ & $\Delta$ \\
\hline \hline 497,0 & 500,7 & 497,2 & 497,1 & 3,6 \\
498,0 & 501,5 & 498,5 & 498,2 & 3,3 \\
499,1 & 502,9 & 499,9 & 499,5 & 3,4 \\
501,6 & $\tilde{\jmath} 06,0$ & 502,8 & 502,2 & 3,8 \\
503,9 & 508,0 & $50 \tilde{\jmath}, 0$ & $亏 04, \bar{\jmath}$ & 3,5
\end{tabular}

Media $\Delta=3,5$

$$
\mathrm{H}=4450
$$

\begin{tabular}{c|c|c||c|c}
$a$ & $b$ & $c$ & $m$ & $\Lambda$ \\
\hline \hline 494,1 & 498,9 & 495,0 & 494,5 & 4,4 \\
494,0 & 500,4 & 497,0 & 495,5 & 4,9 \\
497,3 & 503,4 & 499,6 & 498,4 & 5,0 \\
$\tilde{5} 00,0$ & 506,3 & 503,0 & 501,5 & 4,8 \\
502,5 & 508,3 & 505,1 & $\overline{5} 03,8$ & 4,5
\end{tabular}

Media $\Delta=4,7$

\begin{tabular}{c|c|c||c|c}
$a$ & $b$ & $c$ & $m$ & $\Delta$ \\
\hline 492,8 & 497,3 & 495,0 & 493,9 & 3,4 \\
495,9 & 500,1 & 496,2 & 496,0 & 4,1 \\
496,6 & 504,3 & 501,3 & 500,4 & 3,9 \\
501,8 & 506,7 & 503,2 & 502,5 & 4,2 \\
502,0 & 506,9 & 504,5 & 503,2 & 3,7
\end{tabular}

\begin{tabular}{|c|c|c|}
\hline $\mathrm{H}$ & Media $\Delta$ & $8 \times 10^{7}$ \\
\hline 3350 & div. 2,6 & 57 \\
\hline 3640 & $\gg \quad 3,0$ & 66 \\
\hline 3930 & $》 \quad 3, \tilde{a}$ & 77 \\
\hline 4190 & $\gg \quad 3,9$ & 86 \\
\hline 4450 & $\gg \quad 4,7$ & 104 \\
\hline
\end{tabular}

Media $\Delta=3,9$

Riassunto 
VARIAZIONE DI RESISTENZA DEL MERCURIO ECC.

345

TAv. II.

\section{Amalgama di Bi satura a $190^{\circ}$.}

Diametro del tubo m/m. 0,5 .

Resistenza totale $R^{\prime}=R=\frac{771,5}{228,5} \Omega=3,37 \Omega$

Parte sottoposta al campo $\rho=\frac{1}{2} R^{\prime}=1,69 \Omega$

Deviazione per $\frac{2}{100000} \Omega, d=6,2$

\begin{tabular}{|c|c|c|c|c|}
\hline \multicolumn{5}{|c|}{$\mathrm{H}=3350$} \\
\hline$a$ & $b$ & $c$ & $m$ & $\Delta$ \\
\hline 486,0 & 491,8 & 485,8 & 485,9 & $\check{5}, 9$ \\
\hline 495,2 & 503,0 & 497,8 & 496,5 & $6, \check{o}$ \\
\hline 498,0 & 504,5 & 499,0 & $498, \tilde{0}$ & 6,0 \\
\hline 503,0 & 511,0 & 505,8 & 504,4 & 6,6 \\
\hline 506,7 & 513,8 & 508 & 507,7 & 6,1 \\
\hline
\end{tabular}

\begin{tabular}{|c|c|c|c|c|}
\hline \multicolumn{5}{|c|}{$\mathrm{H}=3640$} \\
\hline 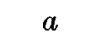 & $b$ & $c$ & $m$ & $\Delta$ \\
\hline & 493,9 & 487,9 & 486,5 & 7,4 \\
\hline & $49 \tilde{0}, 2$ & 488,4 & 488,2 & 7,0 \\
\hline & 504,8 & 500,5 & 497,9 & 6,9 \\
\hline & 509,0 & 503,7 & 501,7 & 7,3 \\
\hline & 513,1 & 508,0 & 506,0 & 7,1 \\
\hline
\end{tabular}

$$
\mathrm{H}=3930
$$

\begin{tabular}{c|c|c||c|c}
$a$ & $b$ & $c$ & $m$ & $\boldsymbol{\Lambda}$ \\
\hline 490,8 & $\tilde{5} 00,1$ & 491,6 & 491,2 & 8,9 \\
492,2 & $\tilde{5} 00,8$ & 492,8 & $492, \tilde{\Xi}$ & 8,3 \\
494,0 & 502,8 & 494,6 & 494,3 & $8, \tilde{5}$ \\
$496, \tilde{5}$ & 504,9 & 497,8 & 496,9 & 8,0 \\
498,1 & $\tilde{5} 07,3$ & 499,3 & 498,7 & 8,6
\end{tabular}

Media $\Delta=8,5$

$$
\mathrm{H}=4450 \text {. }
$$

\begin{tabular}{|c|c|c|c|c|}
\hline & $b$ & $c$ & $m$ & $\Delta$ \\
\hline 86,8 & 496,9 & 487,0 & 486,9 & 10.0 \\
\hline 487,3 & 497,3 & 488,1 & 487,7 & 9,6 \\
\hline 488,7 & 499,8 & 490,6 & 489,6 & 10,2 \\
\hline 493,1 & 504,4 & 496,0 & $494, \overline{0}$ & 9,9 \\
\hline 499,2 & 510,5 & 502,3 & 500,7 & 9,8 \\
\hline
\end{tabular}

Media $\Delta=7,1$

\begin{tabular}{c|c|c||c|c}
$a$ & $b$ & $c$ & $m$ & $\Delta$ \\
\hline 483,0 & 493,9 & 486,0 & 484,5 & 9,4 \\
486,5 & 498,7 & 493,2 & 489,8 & 8,9 \\
494,0 & 506,7 & 501,3 & 497,6 & 9,1 \\
502,2 & 513,9 & 508,1 & 505,1 & 8,8 \\
509,1 & 520,9 & 514,7 & 511,8 & 9,0
\end{tabular}

Media $\Delta=9,0$

Riassunto

\begin{tabular}{c|r|c}
$\mathrm{H}$ & Media $\Delta$ & $8 \times 10^{7}$ \\
\hline 3350 & div. 6,2 & 119 \\
3640 & $\gg 7,1$ & 136 \\
3930 & $\gg 8,5$ & 163 \\
4190 & $\gg 9,0$ & 173 \\
4450 & $\gg 9,9$ & 190
\end{tabular}


Nel quadro che segue riporto i valori delle frazioni $p$ di resistenza dei liquidi cimentati e sottoposte direttamente all'azione del campo, notando contemporaneamente con $\mathrm{D}$ il diametro dei tubi e con $d$ la deviazione dovuta per $\frac{2}{100000}$ di Ohm.

\begin{tabular}{|c|c|c|c|c|}
\hline Liquido & Cimentato & $\mathrm{D}$ & $\rho$ & $d$ \\
\hline & $\mathrm{Hg}$ distillato & $\mathrm{m} / \mathrm{m} \cdot 0,7$ & 1,89 & 6,8 \\
\hline Amalgama di & Bi satura a $12^{\circ}$ & $\gg \quad 0,7$ & 1,80 & 6,2 \\
\hline & $\mathrm{Hg}$ distillato & $\gg \quad 0,5$ & 1,74 & 5,2 \\
\hline Amalgama di & $0,5 \mathrm{Bi}+100 \mathrm{Hg}$ & 0,5 & 1,66 & 6,7 \\
\hline » & $1,0 \mathrm{Bi}+100 \mathrm{Hg}$ & $0, \tilde{5}$ & 1,68 & 6,9 \\
\hline$»$ & Bi satura a $12^{0}$ & 0,5 & 1,66 & 6,4 \\
\hline$\gg$ & $\gg \quad>190^{\circ}$ & 0,5 & 1,68 & 6,2 \\
\hline
\end{tabular}

In questo ultimo quadro, dove $\Delta$ è la deviazione media dovuta al campo, presento $\mathrm{i}$ valori delle $\delta$, 
VARIAZIONE DI RESISTENZA DEL MERCURIO ECC. 347

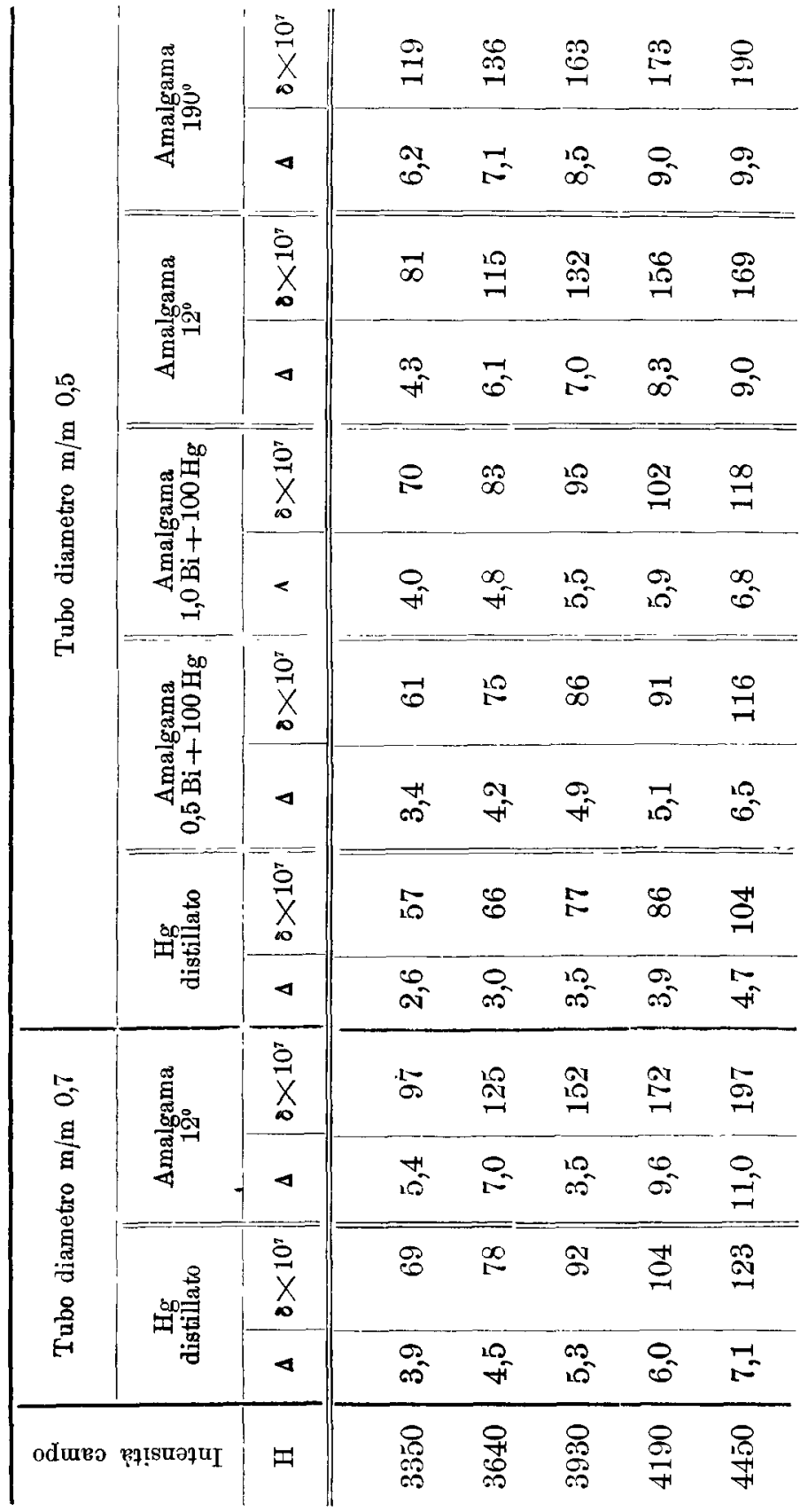


Dai risultati suesposti si deduce:

$1^{0}$ Che il $\mathrm{Hg}$ e le amalgame di $\mathrm{Bi}$ presentano nel campo trasverso, sempre aumento di resistenza.

$2^{0}$ La variazione di resistenza cresce col campo, col diametro del tubo, con la concentrazione delle amalgame.

Ora, se mai l' accusata variazione fosse dovuta a semplice azione elettrodinamica, tale variazione avrebbe dovuto mantenersi inalterata, sia per l' $\mathrm{Hg}$ puro, sia al variare della concentrazione dell' amalgama, per lo stesso tubo, il che $\dot{\theta}$ contro l'esperienza.

È perciò che io reputo potere ammettere che esistono corpi allo stato liquido pei quali, prescindendo da effetti elettrodinamici, la resistenza è suscettibile di variazione nel campo magnetico.

E non è a dirc che le variazioni di resistenza siano da attribuirsi esclusivamente al $\mathrm{Bi}$, perchò, anche nel caso dell' $\mathrm{Hg}$ puro se ne hanno, e dello stesso ordine di grandezza. Quindi si ha motivo di ritenere che la proprietà rilevata, di aversi variazioni di resistenza per effetto del campo magnetico, sia inerente alle resistenze metalliche liquide.

Rendo vive grazie all' illustre prof. Cantone per l'assistenza ed i consigli di cui mi fu largo nei parecchi mesi che durarono le mie ricerche. 